\title{
Cálculo de niveles de energía con los potenciales de Lennard-Jones, Coseno Hiperbólico y Morse utilizando el método matricial y de emparejamiento
}

\section{Calculation of energy levels with Lennard-Jones, Hyperbolic Cosine and Morse potentials using the matrix and pairing method}

\author{
F. Andino ${ }^{1}$ | M. Spilsbury ${ }^{2}$ | M. Reyes $^{3}$ | A. GAlO ${ }^{4}$
}

Recibido: 16 de octubre de 2019/ Aceptado: 21 de junio de 2020

\footnotetext{
${ }^{1}$ Departamento de Física, Universidad Nacional Autónoma de Honduras en el Valle de Sula, San Pedro Sula (Honduras). email: fernando.andino@unah.edu.hn

${ }^{2}$ Departamento de Física, Universidad Nacional Autónoma de Honduras en el Valle de Sula, San Pedro Sula (Honduras). email: michael.spilsbury@unah.edu.hn

${ }^{3}$ Departamento de Física, Universidad Nacional Autónoma de Honduras en el Valle de Sula,

San Pedro Sula (Honduras). email: marco.reyes@unah.edu.hn

${ }^{4}$ Escuela de Física, Facultad de Ciencias, Universidad Nacional Autónoma de Honduras, Tegucigalpa (Honduras), email: alejandrogaloroldan@gmail.com
}

Este trabajo representa un ejercicio académico en el que se comparan dos métodos numéricos; el método matricial y de emparejamiento para la solución de la ecuación unidimensional de Schrödinger con los potenciales de Lennard-Jones, coseno hiperbólico y de Morse. Con ambos métodos, se obtuvieron los niveles de energía y las funciones de onda asociadas con un error bajo, esto comparando las energías calculadas numéricamente con las del potencial coseno hiperbólico las cuales se conocen de forma analítica.

This work represents an academic exercise in which two numerical methods are compared; the matrix and matching method for the solutions to Schrödinger's one-dimensional equation with the Lennard-Jones potential, hyperbolic cosine and Morse. With both methods, the energy levels and associated wave functions are obtained with a low error, this from the comparing between the numerically calculated energies with those of hyperbolic cosine potential which are known analytically.

\section{PALABRAS CLAVES}

Ecuación de Schrödinger, niveles de energía, método matricial, método de emparejamiento.

\section{KEYWORDS}

Schrödinger equation, energy levels, matrix method, matching method. 


\section{I | INTRODUCCIÓN}

$\mathrm{L}$

A ecuación de Schrödinger se puede resolver para muy pocos potenciales de forma analítica, lo cual hace natural el uso de las técnicas numéricas. Este trabajo tiene como propósito proporcionar a los estudiantes de física dos herramientas numéricas muy eficientes para resolver la ecuación de Schrödinger en una dimensión para cualquier potencial, independientemente de su complejidad, por ejemplo el potencial de Lennard-Jones no admite una solución exacta pero si numérica.

Para describir el movimiento de una partícula sometida a una fuerza aplicamos la segunda ley de Newton y resolvemos las ecuaciones respectivas, lo anterior es considerando que la partícula se comporta de forma clásica Eisberg (2002), Griffiths (2016).

La mecánica cuántica proporciona un enfoque muy diferente para el mismo problema, en este caso buscamos la función de onda de la partícula $\Psi(x, t)$, la cual se obtiene resolviendo la ecuación de Schrödinger

$$
i \hbar \frac{\partial \Psi}{\partial t}=-\frac{\hbar^{2}}{2 m} \frac{\partial^{2} \Psi}{\partial x^{2}}+V \Psi
$$

donde $i$ es la unidad imaginaria, $\hbar$ es la constante de Planck dividida entre $2 \pi, m$ es la masa de la partícula de estudio y $V$ es la energía potencial a la que esta sometida la partícula Eisberg (2002), Griffiths (2016). La ecuación de Schrödinger juega un papel análogo a la segunda ley de Newton.

La ecuación de Schrödinger independiente del tiempo se puede escribir así

$$
-\frac{\hbar^{2}}{2 m} \frac{d^{2} \psi}{d x^{2}}+V \psi=E \psi
$$

donde $E$ es la energía total de la partícula Griffiths (2016). Ahora bien, ¿qué es la función de onda? y una vez que la tenemos ¿para qué sirve?, estas son preguntas que surgen de inmediato. La función de onda $\Psi(x, t)$ tiene un carácter inherentemente complejo. No existen instrumentos que midan el valor de la función de onda, ya que es imposible medir con un instrumento físico y real el valor de una cantidad compleja Eisberg (2002).

No se debe considerar un punto débil de la teoría de la mecánica cuántica el hecho que las funciones de onda sean funciones complejas. De hecho lo anterior es deseable ya que nos impide darle a las funciones de onda una interpretación física análoga a las ondas en una cuerda u ondas que se propagan en el agua. Esto nos evita formular preguntas como ¿qué es lo que ondula? y ¿dónde ondula? Eisberg (2002), Griffiths (2016). Las funciones de onda son instrumentos de cómputo que solo tienen significado en el contexto de la teoría de Schrödinger de la que son parte. La función de onda contienen toda la información compatible con el principio de incertidumbre.

Para obtener toda esta información debemos relacionar cuantitativamente $\Psi(x, t)$ con las cantidades dinámicas en la región de la coordenada $x$ en el tiempo $t$ y la probabilidad por unidad de longitud, o densidad de probabilidad $P(x, t)$, ya que la densidad de probabilidad es una cantidad real, mientras que la función de onda es una función compleja Griffiths (2016). Born hizo la asociación en 1928 en la forma de un postulado:

$\mathrm{Si}$, al tiempo $t$ se efectuá una medida para ubicar la partícula asociada con la función de onda $\Psi(x, t)$, entonces la probabilidad $P(x, t) d x$ de que el valor de la coordenada se encuentre entre $x$ y $x+d x$ es

$$
P(x, t) d x=\Psi^{*}(x, t) \Psi(x, t) d x,
$$


donde el símbolo $\Psi^{*}(x, t)$ es el complejo conjugado de $\Psi(x, t)$. Es claro que se debe cumplir que

$$
\int_{-\infty}^{\infty} \Psi^{*}(x, t) \Psi(x, t) d x=1
$$

y de la estadística sabemos que al integrar la densidad de probabilidad en todo el espacio esto debe de dar como resultado la unidad, lo cual es cierto para todo tiempo $t$ Griffiths (2016).

Las funciones de potencial modelan como interactúan los átomos y moléculas, por ejemplo el potencial de Lennard-Jones incluye la repulsión y atracción para un par de átomos neutros, considerándose un potencial realista especialmente para estudiar los gases nobles. La función potencial es

$$
V(r)=4 \varepsilon\left[\left(\frac{\sigma}{r}\right)^{12}-\left(\frac{\sigma}{r}\right)^{6}\right]
$$

donde $\varepsilon$ es la profundidad del potencial, $\sigma$ es la distancia en la que el potencial entre las partículas es cero y r es la distancia entre las partículas Giordano y Nakanishi (2005). El potencial de Morse y coseno hiperbólico modelan la interacción entre moléculas diatómicas, estas son las funciones

$$
\begin{gathered}
V(r)=D_{e}\left[1-e^{-a\left(r-r_{e}\right)}\right]^{2} \\
V(r)=\frac{\hbar^{2}}{2 m} \alpha^{2} \lambda(\lambda-1)\left[\frac{1}{2}-\frac{1}{\cosh ^{2}(\alpha r)}\right]
\end{gathered}
$$

\section{II | MÉTODO MATRICIAL}

Al escribir la ecuación de Schrödinger independiente del tiempo, Ecuación 2, en diferencias finitas utilizando el método de Verlet Vesely (2012) cuya precisión es del tercer orden $\left[O\left(\Delta x^{4}\right)\right]$, se tiene

$$
-\frac{1}{2} \frac{\psi_{i+1}-2 \psi_{i}+\psi_{i-1}}{(\Delta x)^{2}}+V_{i} \psi_{i} \approx E \psi_{i},
$$

y al despejar para $\psi_{i+1}$ se obtiene

$$
\psi_{i+1}=2 \psi_{i}-\psi_{i-1}-2(\Delta x)^{2}\left(E-V_{i}\right) \psi_{i}
$$

Se puede explorar más sobre el método de diferencias finitas en Quarteroni (2007) Quarteroni y Saleri (2007). Por último, reescribimos la ecuación anterior para utilizarla en forma matricial, quedando

$$
\left(\frac{1}{(\Delta x)^{2}}+V_{i}-E\right) \psi_{i}-\frac{1}{2(\Delta x)^{2}} \psi_{i-1}-\frac{1}{2(\Delta x)^{2}} \psi_{i+1}=0
$$

Las condiciones de frontera especifican los valores de $\psi$ en la frontera de la región de estudio, la cual podemos imaginar como una caja, $\psi$ debe ser cero en los extremos del sistema, esto significa que $\psi(0, n)=\psi(M, n)=\psi(m, 0)=\psi(m, N)$, donde el mallado corre desde $m=0$ a $M$ у $n=0$ a $N$. Al evaluar sobre la Ecuación 10 obtenemos un sistema de ecuaciones algebraicas para $\psi(m, n)$, donde hay $M \times N$ valores desconocidos de $\psi(m, n)$ y el mismo número de ecuaciones Giordano y Nakanishi (2005). 
Al evaluar nos quedan matrices que cada vez se vuelven más grandes, veamos un par de ellas:

$$
\begin{aligned}
& \left|\begin{array}{ccc}
-\frac{1}{(\Delta x)^{2}}+V_{2}-E & 0 & 0 \\
-\frac{1}{2(\Delta x)^{2}} & -\frac{1}{(\Delta x)^{2}}+V_{3}-E & -\frac{1}{2(\Delta x)^{2}} \\
0 & -\frac{1}{2(\Delta x)^{2}} & -\frac{1}{(\Delta x)^{2}}+V_{4}-E
\end{array} \| \psi_{3}\right|=\left|\begin{array}{l}
\psi_{2} \\
0 \\
0
\end{array}\right|
\end{aligned}
$$

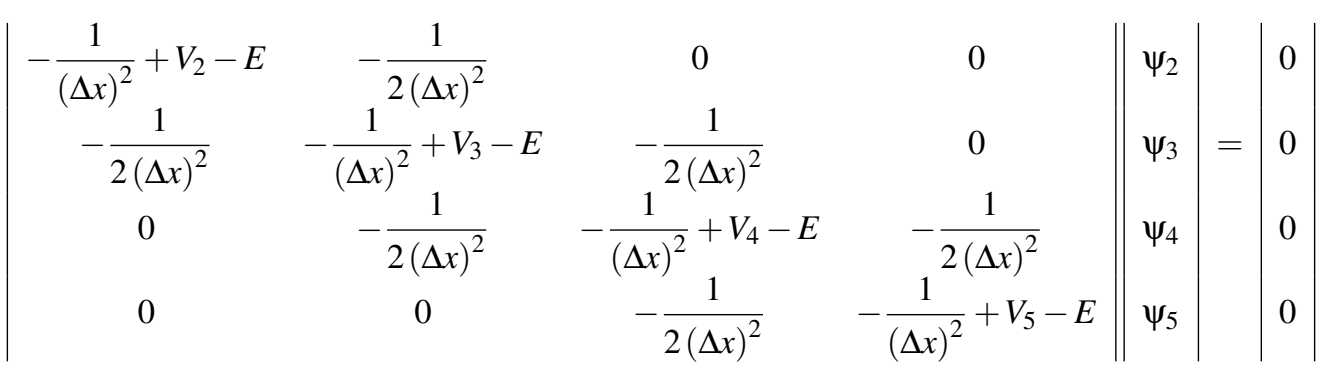

Ahora el problema es encontrar los valores propios. Para calcular estos, existen varias técnicas numéricas, veamos brevemente la teoría al respecto.

Dada una matriz cuadrada $\mathrm{A}$, nos preguntamos como obtener los valores propios. Básicamente estamos interesados en las soluciones de la ecuación $A \vec{x}=\lambda \vec{x}$, la cual es equivalente $(A-\lambda I) \vec{x}=\overrightarrow{0}$, ya que estamos interesados en las soluciones distintas de cero de esta ecuación, la matriz $A-\lambda I$, debe ser singular y por lo tanto, $\operatorname{det}(A-\lambda I)=0$. En principio podemos hallar los valores propios de $A$, si se construye

$$
P(\lambda)=\operatorname{det}(A-\lambda I),
$$

y se hayan los ceros de $P$. La funcíon $P(\lambda)$ se llama el polinomio característico de $A$. En general una matriz de $n \times n$ tendrá un polinomio característico de grado $\mathrm{n}$ y sus raíces son los valores propios de $\mathrm{A}$. El cálculo de ceros de un polinomio es numéricamente desafiante, así que este sencillo procedimiento no es recomendable. Al calcular los valores propios de las matrices asociadas a la ecuación de Schrodinger se obtienen los niveles de energía, estos se calcularon utilizando Octave y a continuación se presenta el Código 1, que primero genera las matrices evaluando la Ecuación 10, luego calcula los valores propios y funciones de onda, y finalmente gráfica las funciones de onda asociadas Giordano y Nakanishi (2005), Gould y Tobochnik (2012).

Los valores propios y por tanto los niveles de energía, el programa los calcula con la librería eig $(A)$.

Código 1: Método matricial.

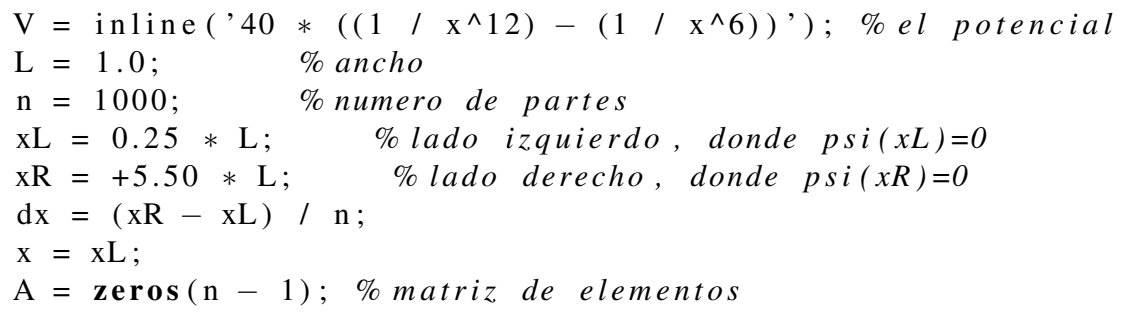




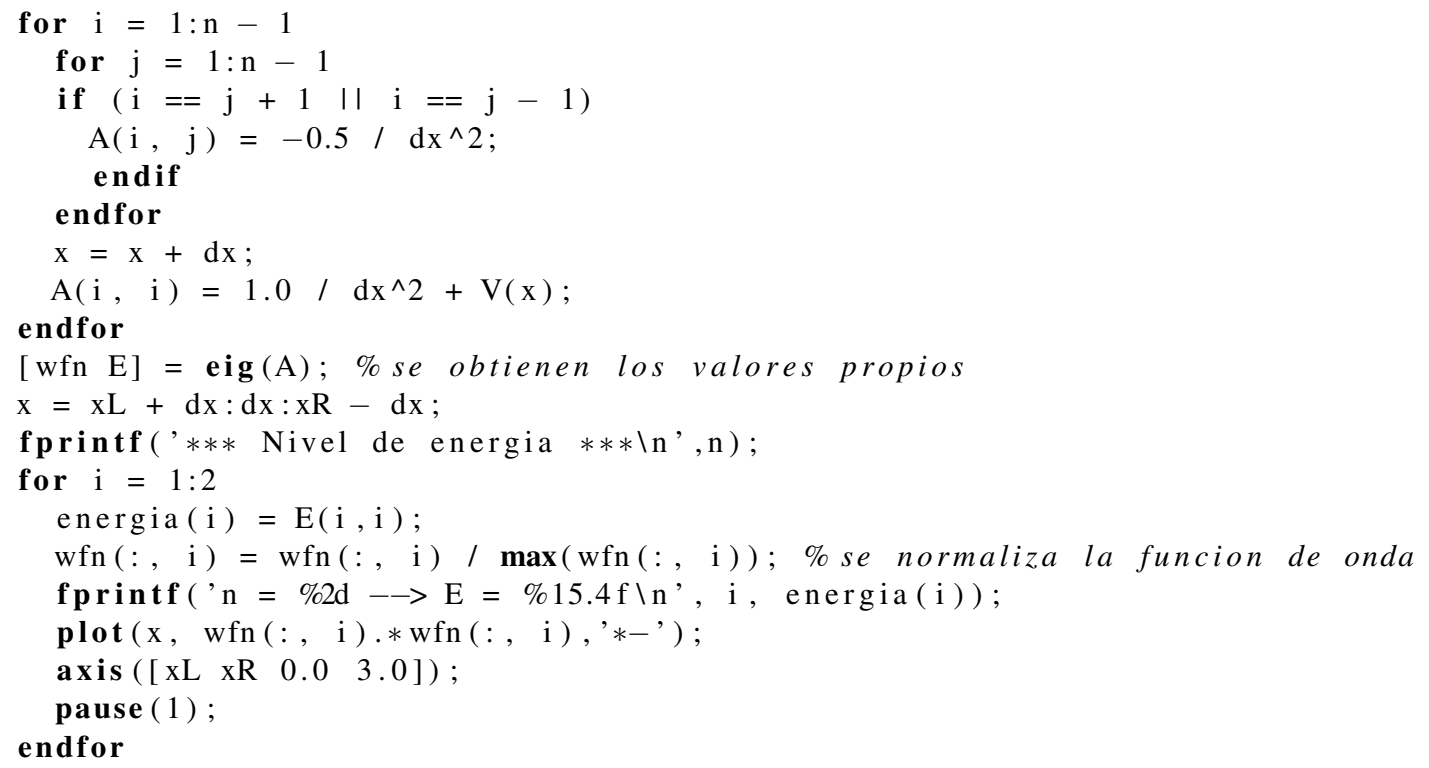

\section{III | MÉTODO DE EMPAREJAMIENTO}

Tomando referencia de Giordano y Nakanishi (2005) Giordano y Nakanishi (2005), el método de emparejamiento (matching) es útil en los casos de potenciales no simétricos, como el de Lennard-Jones, para un sistema de dos átomos, con uno ubicado en $x=0$. Físicamente se espera encontrar con alta probabilidad un estado ligado de un átomo cerca del mínimo potencial. En este estado, la función de onda será cero para valores cercanos a $x=0$ y para valores de $x$ muy grandes; y será significativamente diferente de cero para la región donde $V$ es grande en magnitud y negativa .

Vamos a utilizar nuevamente la ecuación de Schrödinger independiente del tiempo para una partícula moviéndose en una dimensión escrita en la forma de diferencias finitas, Ecuación 9 para desarrollar el código en Octave. En este punto mencionaremos que este desarrollo de diferencias finitas es utilizando el método de Verlet, el cual tiene una precisión del tercer orden $\left[O\left(\Delta x^{4}\right)\right]$; luego desarrollaremos con un pequeño cambio el método de Numerov, el cual tiene una precisión del cuarto orden $\left[O\left(\Delta x^{6}\right)\right]$. Se puede consultar estos métodos en Vesely (2012) Vesely (2012).

Utilizando el método de Verlet y de acuerdo a Giordano y Nakanishi (2005) Giordano y Nakanishi (2005):

Consideremos el calculo de $\psi$ usando Ecuación 9, comenzando en la región donde $\psi$ es muy pequeña (p. ej. valores grandes y pequeños de $x$ ) y siguiendo hacia el medio. Por lo que realizaremos dos integraciones, una comenzando en el punto más a la izquierda de la Figura 1, en el punto $x=x_{I}$. En este punto, se asigna $\psi=0$ y un valor de $d \psi / d x$ muy pequeño. Entonces al usar la Ecuación 9 se obtiene $\psi$ en $x_{I}+\Delta x, x_{I}+2 \Delta x$, etc., así se determina $\psi$ con cada incremento del valor de $x$. A esta función se le llama $\psi_{I}$, porque se obtiene por integración desde la región más a la izquierda. Este procedimiento se repite comenzando en el punto $x=x_{D}$, el más alejado a la derecha de la Figura 1 . Nuevamente, en este punto se asigna $\psi=0$ y un valor de $d \psi / d x$ pequeño, antes de usar la Ecuación 9 otra vez. Entonces se integra hacia valores decrecientes de $x$, y así obtener la función 


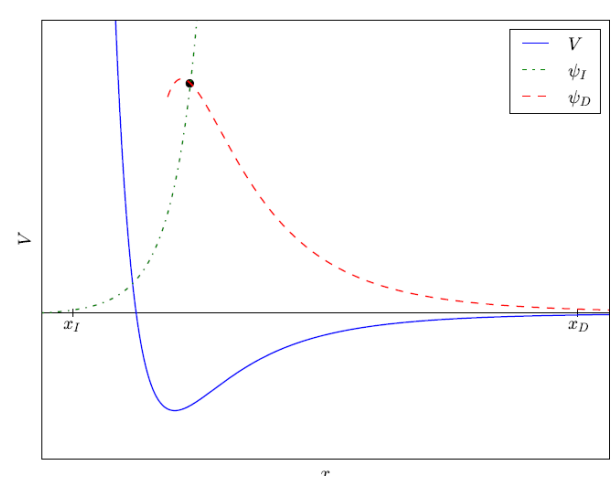

Figura 1: Ilustración esquemática del método de emparejamiento para resolver la ecuación de Schrödinger. La curva sólida muestra el potencial, mientras que las curvas discontinuas muestran funciones de onda (hipotéticas), iniciando la integración desde la izquierda $\left(\psi_{I}\right)$ y desde la derecha $\left(\psi_{D}\right)$. En este caso, las dos funciones de prueba no coinciden con suavidad, y por lo tanto no se pueden combinar para dar una solución aceptable de la ecuación de Schrödinger Giordano y Nakanishi (2005).

llamada $\psi_{D}$. La idea es combinar las funciones $\psi_{I}$ y $\psi_{D}$ para obtener la función de onda en todo el rango.

La función aceptable debe ser suave y continua. Esto indica que $\psi$ y sus derivadas deben ser continuas. Entonces la combinación de las dos funciones $\psi_{I}$ y $\psi_{D}$ para que sea una función de onda aceptable, se deben intersecar en algún punto (función $\psi$ continua) y deben tener la misma pendiente en el punto de intersección (derivada $d \psi / d x$ continua).

Generalmente $\psi_{I}$ o $\psi_{D}$ o ambas se pueden multiplicar por un factor constante para que sean iguales en el punto de coincidencia y aún obtener una solución a la ecuación de Schrödinger, este punto generalmente es conveniente y numéricamente aconsejable que sea cercano al mínimo del potencial, ya que la función de onda tendrá un valor grande allí. Esto todavía nos deja con el requisito de que las derivadas sean iguales en el punto de coincidencia. El único parámetro ajustable restante es la energía. Para la mayoría de los valores de $E$ la Figura 1 nos muestra esta situación, donde las derivadas $d \psi_{L} / d x$ y $d \psi_{R} / d x$ no coinciden. Por suerte, es posible ajustar el valor de $E$ para hacer coincidir estas derivadas. Cuando esto sucede, la combinación de $\psi_{I}$ y $\psi_{D}$ nos da la función de onda correcta, y el valor asociado de $E$ es la energía del nivel.

Por esta razón es conocido como método de emparejamiento (matching). La rutina de búsqueda a utilizar debe dar una correspondencia cada vez más exacta de las derivadas $d \psi_{I} / d x$ y $d \psi_{D} / d x$, y por lo tanto en cada iteración mejores estimaciones para la función de onda y la energía. En primer lugar, debido a que se calculará $\psi(x)$ desde ambos extremos del sistema, debemos tener valores iniciales de las funciones de onda y sus derivadas en estos punto. Es decir, necesitamos comenzar con $\psi_{I}\left(x_{I}-\Delta x\right)$, $\psi_{I}\left(x_{I}\right), \psi_{D}\left(x_{D}\right)$ y $\psi_{D}\left(x_{D}+\Delta x\right)$; donde los dos valores fuera del intervalo $\left(x_{I}, x_{D}\right)$ son necesarios para empezar con la iteración de la Ecuación 9. Estos valores se escogen generalmente de tal forma que las pendientes iniciales sean muy pequeñas, por ejemplo se puede tomar $\psi_{I}\left(x_{I}-\Delta x\right)=-0.0001 \Delta x$ Giordano y Nakanishi (2005). Por conveniencia, podemos elegir el mismo valor en ambos extremos ya que se aplicará el factor de escala de manera diferente para coincidir con los valores de $\psi_{I}$ y $\psi_{D}$ en el punto central. En segundo lugar, y más importante, debemos tener una estrategia eficiente de variar el 


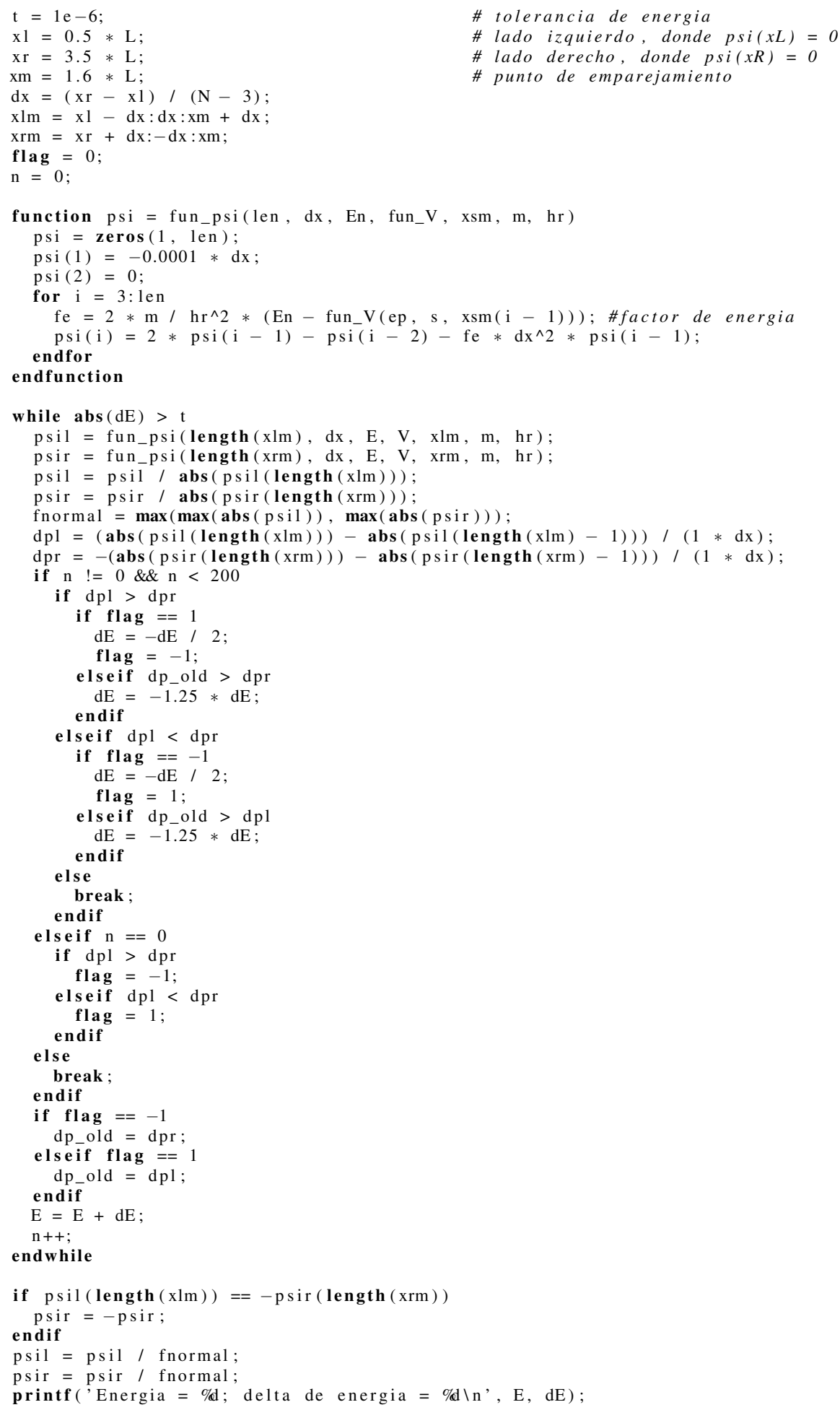




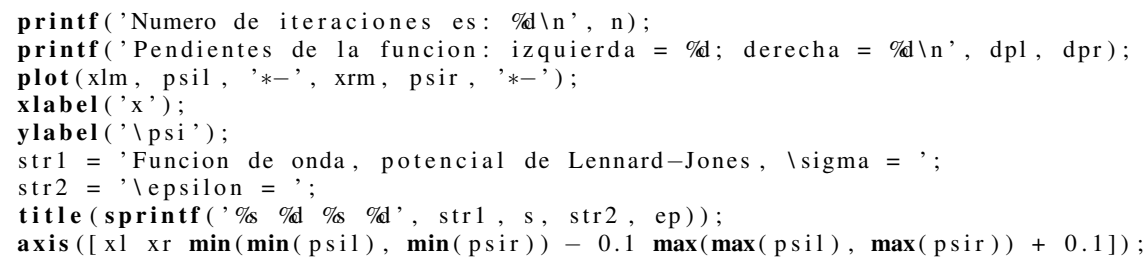

\section{I RESULTADOS}

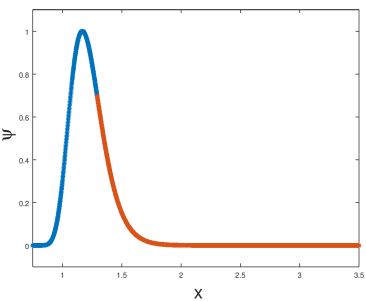

(a) $n=1$

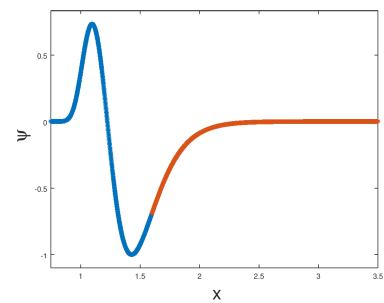

(b) $n=2$

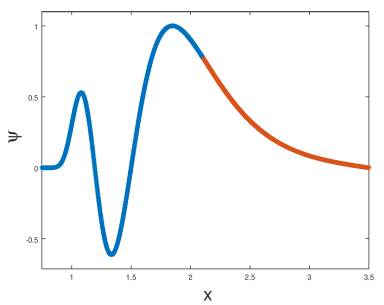

(c) $n=3$

Figura 2: Función de onda para el potencial de Lennard-Jones, con $\sigma=1, \varepsilon=100$; utilizando el método de emparejamiento. Se muestran los 3 primeros niveles de energía.

\begin{tabular}{c|c|c}
\hline$n$ & $E_{n}$ matricial, & $E_{n}$ emparejamiento \\
\hline 1 & -66.2694 & -66.2671 \\
2 & -22.9814 & -22.9822 \\
3 & -4.1324 & -4.1321 \\
4 & 1.1160 & 1.1186 \\
\hline
\end{tabular}

Tabla 1: Niveles de energía, en unidades reducidas, con el potencial de $L J$, con $\varepsilon=100$ y $\sigma=1$.

Ahora se reportan los resultados obtenidos para cada potencial. Se calculan los niveles de energía con los dos métodos, matricial y emparejamiento, variando los valores de los parámetros que caracterizan los potenciales, en cada caso se presentan las gráficas de las funciones de onda correspondientes.

\section{Potencial de Lennard-Jones}

Como ya sabemos el potencial de Lennard-Jones esta caracterizado por los dos parámetros $\varepsilon$ y $\sigma$, y está dado por la Ecuación 5 Los primeros niveles de energía que se obtuvieron se muestran en la Tabla 1

Los niveles de energía para el potencial de Lennard Jones no pueden ser calculados de manera exacta, solamente se pueden obtener de forma numérica, en Giordano y Nakanishi (2005) Giordano y Nakanishi (2005) se calculó la energía base para los parámetros $\varepsilon=10$ y $\sigma=1$, la energía reportada es $E_{1}=-1.969$ esta energía la vamos a tomar de referencia. Con el método matricial obtuvimos $E_{1}=-1.8899$ el cual difiere un $4.02 \%$ con respecto al de Giordano, con el método de emparejamiento 


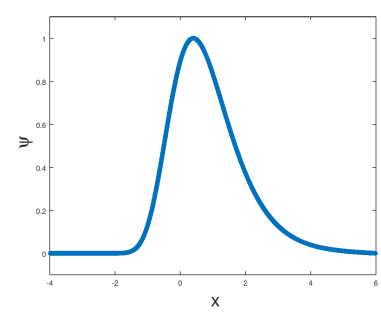

(a) $n=1$

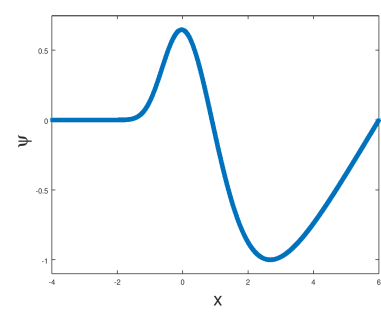

(b) $n=2$

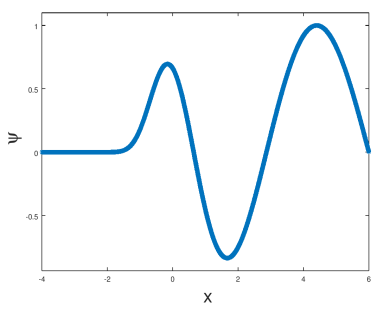

(c) $n=3$

Figura 3: Función de onda para el potencial de Morse con $D_{e}=1.5, r_{e}=0.06$ y a $=1$; utilizando el método matricial. Se muestran los 3 primeros niveles de energía.

\begin{tabular}{c|c|c}
\hline$n$ & $E_{n}$ matricial, & $E_{n}$ emparejamiento \\
\hline 1 & 0.7410 & 0.7410 \\
2 & 1.5120 & 1.5120 \\
3 & 1.9583 & 1.9584 \\
4 & 2.6303 & 2.6304 \\
\hline
\end{tabular}

Tabla 2: Niveles de energía, en unidades reducidas, con el potencial de Morse, con $D_{e}=1.5, r_{e}=0.06$ y $a=1$.

se obtuvo $E_{1}=-1.8941$ el cual difiere $3.80 \%$.

La Figura 2 muestra como cambia $\psi$ versus $x$, el cambio de color muestra el punto donde ocurre el emparejamiento.

\section{Potencial de Morse}

Tomando $D_{e}=1.5, r_{e}=0.06$ y $a=1$ en la Ecuación 6 se obtienen los niveles de energíamostrados en la Tabla 2

De acuerdo a Reyes (2019) Pagoada (2019), la energía base, o energía mas baja, para el potencial de Morse

$$
V=2\left(1-e^{-r}\right)^{2}
$$

calculado mediante el método de caminata aleatoria es $E_{1}=0.8541$, vamos a tomar este resultado como referencia para comparar con los métodos de este trabajo. Con el método matricial obtuvimos $E_{1}=0.8750$ este resultado difiere $2.45 \%$ con respecto al de Reyes, con el método de emparejamiento $E_{1}=0.8749$ difiere en $2.44 \%$ también.

Además si se utilizan $D_{e}=200$ y $a=0.05$, y utilizando la solución aproximada

$$
E_{n}=a \sqrt{2 D_{e}}\left(n+\frac{1}{2}\right)-\frac{a^{2}}{2}\left(n+\frac{1}{2}\right)^{2},
$$

como se expone en Okock y Burns (2015), se obtienen los niveles de energíamostrados en la Tabla 3 


\begin{tabular}{c|c|c|c}
\hline$n$ & $E_{n}$ matricial & $E_{n}$ emparejamiento & $E_{n}$ aproximado \\
\hline 1 & 0.4997 & 0.4997 & 0.4997 \\
2 & 1.4972 & 1.4971 & 1.4972 \\
3 & 2.4922 & 2.4922 & 2.4922 \\
4 & 3.4848 & 3.4848 & 3.4847 \\
\hline
\end{tabular}

Tabla 3: Niveles de energía, en unidades reducidas, con el potencial de Morse, con $D_{e}=200$ y $a=0.05$.

\begin{tabular}{c|c|c|c}
\hline$n$ & $E_{n}$ matricial, & $E_{n}$ emparejamiento & $E_{n}$ teórico \\
\hline 1 & -1.5000 & -1.5000 & -1.5000 \\
2 & 1.0000 & 1.0000 & 1.0000 \\
3 & 2.5000 & 2.5000 & 2.5000 \\
4 & 3.0184 & 3.0185 & 3.0000 \\
\hline
\end{tabular}

Tabla 4: Niveles de energía, en unidades reducidas, del potencial coseno hiperbólico. $\alpha=1$ y $\lambda=4$.

\section{Potencial de coseno hiperbólico}

De acuerdo a Tao Pang Pang (2010), la solución analítica para el potencial de la Ecuación 7 es:

$$
E_{n}=\frac{\hbar^{2}}{2 m} \alpha^{2}\left[\frac{\lambda(\lambda-1)}{2}-(\lambda-1-n)^{2}\right]
$$

Tomando $\alpha=1$ y $\lambda=4$, los niveles de energía se muestran en Tabla 4

La tabla anterior muestra lo que predicen el método matricial y emparejamiento de los niveles de energía, y se muestra al mismo tiempo cuales son los valores exactos para el potencial coseno hiperbólico. Los valores son muy parecidos pero como se menciono anteriormente, el método de emparejamiento trabaja mejor con potenciales no simétricos Giordano y Nakanishi (2005).

Para poder comparar el tiempo de calculo en la obtención de los niveles de energía con cada método numérico y para los diferentes potenciales se uso la función cputime de Octave. Se observó que para el mismo número de puntos en el intervalo de calculo, al método matricial le toma mucho más tiempo en los cálculos, pero con la ventaja que calcula varios niveles de energía a la vez, mientras que el método de emparejamiento solo calcula un nivel de energía por vez. Respecto al método de emparejamiento, comparando el método de Verlet y Numerov, el de Numerov le toma casi el doble del tiempo que con el de Verlet y no hay diferencia en cuanto a la precisión.

De acuerdo a Giordano y Nakanishi (2005) Giordano y Nakanishi (2005) el método matricial se puede implementar para resolver la ecuación de Schrödinger en más dimensiones, el método de emparejamiento se puede utilizar solo en una dimensión. 


\section{I CONCLUSIONES}

1. Al resolver la ecuación de Schrödinger unidimensional independiente del tiempo utilizando cualquiera de los potenciales de estudio, ya sea con el método matricial o el de emparejamiento y al comparar los resultados, estos son muy similares. En el caso del potencial coseno hiperbólico, al compararlos con la solución exacta, ambos dan el mismo porcentaje de error (alrededor del $0.7 \%$ ).

2. Los tiempos de calculo son muy diferentes. Considerando los mismos parámetros como ser el intervalo $x$ de evaluación de la función de onda, el $\Delta x$ entre puntos consecutivos y los demás parámetros de las funciones de potencial, al método matricial le toma mucho mas tiempo pero calcula los niveles de energía para varios estados a la vez, como se menciono antes. Para el ejemplo del potencial de Lennard-Jones, tomando 2500 puntos para el intervalo entre $x=0.75$ y $x=3.5$, con el método de matricial el tiempo de calculo fue aproximadamente 4 veces el tiempo que le tomó calcular al método de emparejamiento los 4 valores de energía. Esto se debe a que el método matricial calcula $n$ valores propios ( $n$ valores de energía permitidos) correspondiente a los $n$ puntos del $\Delta x$ en el intervalo de análisis.

3. Se observó que la precisión de los valores de energía en el método de emparejamiento dependen, además de los datos de entrada mencionados anteriormente, la energía inicial, tolerancia de energía y el punto donde se cortan las funciones de onda $\psi_{I}$ y $\psi_{D}$. Para el caso de los potenciales con valores teóricos o aproximaciones de energía, cuando la función $\psi_{I}$ se corta con $\psi_{D}$ más cerca del final del último pico de la función de onda (ver Figura 2), más exacto era el valor de energía encontrado con dicho método. El tiempo de calculo con este método también dependen en gran medida de los parámetros mencionados.

4. En el caso del método de emparejamiento utilizando el potencial coseno hiperbólico, se comparó la solución dado por el método de Verlet y de Numerov para $n=3$. No se observó diferencia en cuanto a precisión para una determinada tolerancia en energía, pendiente en el punto de emparejamiento o cambio en el $\Delta x$ de separación de puntos consecutivos. La diferencia se dio en el tiempo de calculo, siendo en el método de Numerov alrededor de 2.5 veces el tiempo en el método de Verlet. Al cambiar la tolerancia de 1e-6 a 1e-7 con cualquiera de los dos métodos el tiempo aumenta en promedio 1.5 veces.

5. El método matricial muestra buen desempeño para resolver la ecuación de Schrödinger para cualquier tipo de potencial, en cambio el de emparejamiento, funciona mejor para potenciales no simétricos Giordano y Nakanishi (2005).

\section{REFERENCIAS}

Eisberg, R. M. (2002). Fundamentos de física moderna. Limusa.

Giordano, N. J., y Nakanishi, H. (2005). Computational physics. Pearson, 2nd edition.

Gould, H., y Tobochnik, J. (2012). An introduction to computer simulation methods: Applications to physical systems. CreateSpace Independent Publising Platform.

Griffiths, D. J. (2016). Introduction to quantum mechanics. Cambridge University Press.

Okock, P., y Burns, T. (2015). A matrix method of solving the schrodinger equation (Tesis Doctoral no publicada). African Institute for Mathematical Sciences.

Pagoada, M. A. R. (2019, Junio). Solución de la ecuación de schrödinger con caminata aleatoria. Revista de la escuela de física, VII, 45-51.

Pang, T. (2010). An introduction to computational physics. Cambridge University Press.

Quarteroni, A., y Saleri, F. (2007). Cálculo científico con matlab y octave. Springer Milan. Descargado de https://books.google.es/books?id=Vfo-S7Fj7i4C 
Vesely, F. (2012). Computational physics: An introduction. Springer US. Descargado de https://books.google .es/books? id=CjrUBwAAQBAJ 